\title{
Algorithm for synchronization of electroporation pulse delivery with electrocardiogram
}

\author{
B. Music ${ }^{1}$, T. Jarm' ${ }^{1}$, F. Jager ${ }^{2} \&$ D. Miklavčič ${ }^{1}$ \\ 'University of Ljubljana, Faculty of Electrical Engineering, Slovenia. \\ ${ }^{2}$ University of Ljubljana, Faculty of Computer and Information Science, \\ Slovenia.
}

\begin{abstract}
Electrochemotherapy is a local treatment of tumors in which high voltage electric pulses are used for permeabilization of cell membrane (electroporation) thus enabling increased entry of chemotherapeutic molecules into tumor cells. Application of electroporation pulses to tumors located close to the heart muscle could lead to fibrillation of the heart if the pulses are delivered during the vulnerable period of the heart or if the pulses coincide with heart arrhythmias due to lowered threshold for fibrillation. We present an algorithm based on the analysis of a single-lead electrocardiogram. The algorithm enables safe use of electrochemotherapy by allowing the electroporation pulses to be delivered only outside the vulnerable period and by preventing generation of the pulses in the presence of arrhythmias. The algorithm synchronizes delivery of electroporation pulses with normal heartbeats in real time. The performance of the algorithm was evaluated using 13 records of the European Society of Cardiology ST-T Database. Performance measures of the algorithm were calculated based on beatby-beat comparison of the results of the algorithm with true heart beat annotations of signals of the database. In further studies we plan to implement the algorithm in instruments for clinical electroporation. Thus the usefulness of electrochemotherapy will be extended due to higher level of safety for patients.
\end{abstract}

\section{Introduction}

Cell membrane electropermeabilization or electroporation (EP) is a method in which high voltage electric pulses are used to increase transiently the 


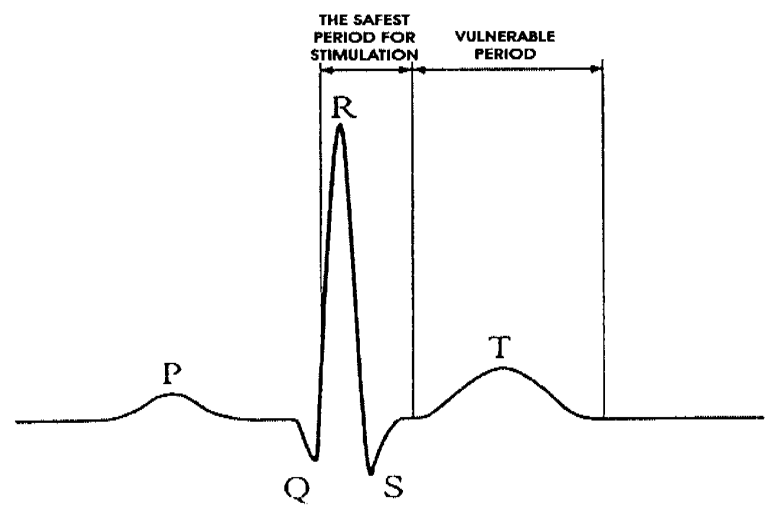

Figure 1: ECG of one heart beat. During the vulnerable period the conduction pathway is still partially refractory, so that the excitation generated by stimulation can propagate in only one direction and therefore induce reentry. The ventricles are not excitable during the QRS period. This is the safest period in ECG for electrical stimulation [7].

permeability of cell membrane, which has important practical applications. One of them is electrochemotherapy (ECT) - the combined treatment in which the injection of chemotherapeutic agent is followed by application of EP pulses locally to the tumor. Electroporated cell membrane allows increased delivery of the drug molecules into the cell. ECT has been used successfully for treatment of various tumors in different animal tumor models and in different tumors in humans [1-4]. In these studies typical ECT protocol involved eight EP pulses with amplitude approximately $1000 \mathrm{~V}$, duration $100 \mu \mathrm{s}$, repetition frequency 1 $\mathrm{Hz}$, and inter-electrode distance $8 \mathrm{~mm}$.

Although the number of clinical trials involving ECT is increasing, some issues concerning the therapy remain open. One of them is the determination of optimal EP pulse parameters to assure successful treatment of tumors with minimal side effects. EP pulses induce transient lesions in areas in direct contact with the electrodes [5]. Muscle contractions in immediate vicinity of the electrodes are responsible for acute pain in local area around the tumor [6]. Contraction of muscles during EP may also be problematic when tumors are located close to the heart muscle. Although none of such complications have been recorded so far, ECT may be harmful for the functioning of the heart because application of EP pulses to such tumors could lead to fibrillation of the heart [7].

Fibrillation of the heart can be induced if the amplitude of the externally applied electric pulses in a part of the heart is greater than the threshold level for fibrillation. The heart is especially susceptible to induction of fibrillation in the so-called vulnerable period (Figure 1). In the ventricles, the non-uniformity in recovery of excitability is maximal preceding the apex of the $\mathrm{T}$ wave in electrocardiogram (ECG). At this time, electrical stimulation elicits an excitation wave that encounters some regions of the heart fully recovered, other regions 
partially recovered, and some regions still absolutely refractory. Propagation of an electrically induced wavefront can thereby be initiated preferentially in certain directions, thus setting the stage for the so-called multiple reentry - the electrophysiological basis of ventricular fibrillation [7]. The heart is thus especially susceptible to external stimulation during the vulnerable period.

Various conditions can damage the heart's conduction system and thereby disturb rhythmical beating of the heart. The term arrhythmia in general refers to any abnormality in heart rhythm. The electrical properties of the heart are changed during appearance of different heart arrhythmias. Conditions for the onset of fibrillation are thus affected; the heart becomes significantly more susceptible to external stimuli [7]. The heart arrhythmias commonly encountered include bradycardia, tachycardia, sinus arrhythmia, extrasystoles of supraventricular or ventricular origin, and atrial or ventricular flutter and fibrillation [8]. In addition to possibility of induction of fibrillation during normal heart beating, EP pulses coinciding with heart arrhythmias could also elicit fibrillation due to significantly decreased threshold level for fibrillation [7]. This potential danger is most significant after a premature response, the extrasystole.

This study is the first step in developing an efficient algorithm, which will enable safe use of EP pulses. The algorithm is based on a simple QRS detection technique and can be used on-line. The algorithm allows EP pulses to be delivered only outside the vulnerable period of each heart beat (Figure 1) and only if no significant variation in the heart rate or R-R interval (indicator of possible arrhythmia) is present in the ECG signal.

\section{Methods}

\subsection{The algorithm}

The algorithm for synchronization of EP pulses with ECG can be split into two major parts (Figure 2). During learning process certain threshold parameters are automatically set according to characteristics of the particular ECG signal. The learning process enables the use of the whole algorithm on different ECG signals originating from the same lead. The decision-making process uses these threshold parameters to detect QRS complexes and to evaluate variations in R-R interval. Based on this, a decision is made whether the EP pulse can be delivered or not. A pulse is delivered if the QRS complex was correctly identified and if the current heart rate does not deviate significantly from the normal heart rate.

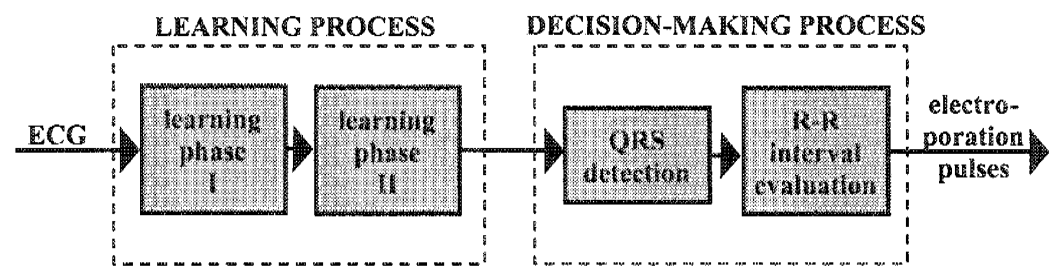

Figure 2: The structure of the developed algorithm. 
Finding the QRS complex is essential for every computer-based ECG analysis. Once the QRS complex was identified, a more detailed examination of ECG signal, including the heart rate, can be performed. However, detection of the QRS complex is often difficult to achieve due to various sources of noise contamination, such as baseline drifts, motion artifacts and muscular activity [9]. Furthermore, morphological differences in the ECG waveforms increase the complexity of QRS detection due to heterogeneity in the QRS waveform and the difficulty in differentiating the QRS complex from tall-peaked $\mathrm{P}$ or $\mathrm{T}$ waves.

The algorithm described here is built for an ECG lead with high dynamics within QRS complex in comparison to other parts of ECG signal. Based on this requirement the standard leads $\mathrm{V}_{4}$ and II appeared to be the most suitable. The signals used in this study all belong to a chest lead $\mathrm{V}_{4}$.

\subsubsection{Learning phase I}

This phase was 20 seconds long and was divided into 20 one-second subintervals. Within each of the subintervals the local maximum slope (MS) according to eqns (1-3) and local maximum amplitude (MA) of the signal was determined. Five largest and five smallest MS and MA values were neglected and the average MS and average MA values were calculated from the remaining 10 values of each parameter. These averaged values were used in calculation of threshold values for further steps of ECG signal analysis.

\subsubsection{Learning phase II}

In this phase QRS complexes were searched for according to the procedure described in subsection 2.1.3. In this phase eleven successive QRS complexes were found, ten $(n)$ R-R intervals evaluated, and their average calculated. This average value $\left(\overline{R R_{n}}\right)$ was used as the measure of the initial "normal" heart rate.

\subsubsection{QRS detection}

Decision rules for EP pulse delivery were based on QRS complex detection. The QRS slope is a popular signal feature used to locate the QRS complex in many detectors [9-12]. A real time derivative algorithm that provides slope information is straightforward to implement but a derivative tends to amplify the undesirable higher frequency noise components. Various abnormal QRS complexes with large amplitudes and long durations can be missed in a purely derivative approach because of relatively low QRS slopes. Therefore QRS slope alone is insufficient for accurate QRS detection. To achieve reliable performance, one has to extract other parameters from the signal such as the amplitude, the width, the R-R interval or the QRS energy [10]. The QRS complex detector used in our work is an adaptation of the detector described in [9]. This detector uses the signal's first and second derivative in its decision rules. However, in order to improve the performance of the algorithm, we added some new features. Detection of QRS complex was based on the following three steps:

1) For each sample of the on-line recorded ECG the measures of the first and the second derivative $(Y 1(n)$ and $Y 2(n)$ respectively) were estimated as: 


$$
\begin{gathered}
Y 1(n)=A B S[X(n+1)-X(n-1)], \\
Y 2(n)=A B S[X(n+2)-2 \cdot X(n)+X(n-2)],
\end{gathered}
$$

where $X(i)$ were the measured sample values of ECG. These two values were scaled and summed thus giving a composite measure of the slope at the $n$-th sample, $Y(n)$ (Friesen [9]):

$$
Y(n)=1,3 \cdot Y 1(n)+1,1 \cdot Y 2(n) .
$$

2) $Y(n)$ was compared to the threshold value for the slope, which was empirically set to $1 / 7$ of the average MS value determined during the learning phase I. If $Y(n)$ was greater than or equal to the threshold value for twelve consecutive samples (a condition stricter than that in the algorithm from [9]), the particular set sample points was very likely to be located on a QRS complex.

3) If the absolute amplitude of the middle six samples form the set of twelve samples defined above were also all above the threshold value for the amplitude, the QRS had been found. The threshold value for the amplitude was empirically chosen as $40 \%$ of the average MA found in learning phase I. By applying the threshold value for the absolute amplitude, we prevented the algorithm from mistaking some other part of the signal having relatively high values of the slope (such as a T-wave or an extrasystole) for a QRS complex.

\subsubsection{R-R interval evaluation}

We used the moving average of ten most recent $\mathrm{R}-\mathrm{R}$ intervals $\left(\overline{R R_{n}}\right)$ as a measure of current "normal" heart rate, starting with the average R-R interval found during learning phase II and therefore according to eqn (4):

$$
\overline{R R_{n}}=0.1 \cdot\left(R R_{n-9}+R R_{n-8}+\ldots+R R_{n}\right),
$$

where $R R_{n}$ is the size of the most recent R-R interval, However, before the most recent $\mathrm{R}-\mathrm{R}$ interval $\left(R R_{n}\right)$ was accepted for calculation of new $\overline{R R_{n}}$, it had to be within the acceptable limits around the previous average value $\overline{R R_{n-1}}$, that is, if the following condition was satisfied:

$$
0.85 \cdot \overline{R R_{n-1}} \leq R R_{n} \leq 1.15 \cdot \overline{R R_{n-1}} .
$$

If the above condition was not satisfied, current $R R_{n}$ was not included in calculation of the current $\overline{R R_{n}}$ and the previous value $\left(\overline{R R_{n-1}}\right)$ was kept as the measure of current "normal" heart rate. The moving average value was updated only when the first next R-R interval satisfying eqn (5) was encountered.

By this simple procedure we avoided abrupt changes in heart rate from being included in the measure of current normal heart rate. Abrupt changes may occur for numerous reasons, but in our case the possibility of the onset of various arrhythmias was of predominant concern. So, even when a QRS complex was found by the QRS detector, our algorithm would not allow an EP pulse to be 
delivered if deviation of the current $\mathrm{R}-\mathrm{R}$ interval from $\overline{R R_{n}}$ was greater than $15 \%$ of the current normal heart rate, eqn (5).

\subsection{Evaluation of the algorithm}

\subsubsection{ECG database}

We evaluated performance of the algorithm using ECG records from the European Society of Cardiology ST-T Database (ESC DB) [13]. This database includes 90 two-hour ambulatory recordings. Each record from this database comprises a two-channel ECG signal and an annotation file containing information about the type of each heart beat. We used 13 signals (see Table 1) from the pool of 50 records of the ESC DB, which are publicly available on Physionet web site [14]. The records chosen were those recorded from the chest unipolar lead $\mathrm{V}_{4}$ and those not containing transient non-ischemic ST-segment episodes due to shifts in the mean electrical axis of the heart.

\subsubsection{Performance metrics for evaluation of QRS complex detection}

The following scores were calculated for each record: $N$ (total number of QRS complexes); $T P=$ true positives (the number of QRS complexes correctly detected); $F N=$ false negatives (the number of missed $\mathrm{QRS}$ complexes); $F P=$ false positives (the number of false QRS detections). Based on these scores, some standard performance measures of the algorithm were calculated:

Sensitivity:

$$
\begin{array}{lrl}
\text { Sensitivity: } & S e(\%)=\frac{T P}{N} \cdot 100, \\
\text { Positive predictivity: } & +P(\%)=\frac{T P}{T P+F P} \cdot 100,
\end{array}
$$$$
\text { Detection error rate: } \quad D E R(\%)=\frac{F P+F N}{N} \cdot 100 \text {. }
$$

\subsubsection{Performance metrics for evaluation of EP pulse delivery}

The following scores were calculated for each record: $N_{p}$ (total number of normal QRS complexes); $T P_{p}=$ true positives for pulses (the number of EP pulses delivered at correctly detected normal QRS complexes); $F N_{p}=$ false negatives for pulses (the number of correctly detected normal QRS complexes where no EP pulse was delivered); $F P_{p}=$ false positives for pulses (the number of EP pulses delivered in absence of correctly detected normal QRS complex). Based on these scores, the following performance measures were calculated:

$$
\begin{array}{lc}
\text { Sensitivity: } & S e_{p}(\%)=\frac{T P_{p}}{N_{p}} \cdot 100, \\
\text { Positive predictivity: } & +P_{p}(\%)=\frac{T P_{p}}{T P_{p}+F P_{p}} \cdot 100, \\
\text { Pulse delivery error rate: } & P D E R(\%)=\frac{F P_{p}+F N_{p}}{N_{p}} \cdot 100 .
\end{array}
$$




\subsection{Programming}

The algorithm was implemented in ANSI C programming language because it enables easy assembler implementation in real time. Evaluation of the algorithm was performed using the MATLAB package (Mathworks Inc.).

\section{Results and discussion}

Figure 3 presents an example of performance of our algorithm. It can be concluded that EP pulses would not be delivered in the case of extrasystoles. This was due to the fact that extrasystoles did not satisfy the criteria for a valid QRS complex (subsection 2.1.3). Even if they did, the R-R interval for extrasystoles did not satisfy the condition for normal heart rate (subsection 2.1.4). It is important to note that EP pulses would not be delivered at normal QRS complexes following the extrasystoles, again due to their R-R intervals, in order to increase the safety margin after the abnormality in functioning of the heart. The results of algorithm evaluation are summarized in Tables 1 and 2 .

The algorithm for synchronization of EP pulse delivery with ECG reliably detected QRS complexes in all 13 signals of chest lead $V_{4}$ from the ESC DB, which where used for testing. For each correctly identified heartbeat and if no abnormalities were detected in the heart rate the algorithm generated an EP pulse. The performance measures for an ideal QRS detector would be $S e=$ $100 \%,+P=100 \%$, and $D E R=0 \%$ and our algorithm approached these values at a degree similar to that of some other detectors with comparably simple algorithms, e.g. [15] (see Table 1). It should be noted that the records with poorest results of the algorithm (large $D E R$ ) contained some features, which our prototypic algorithm was not designed to deal with. These features included: very unstable $\mathrm{R}$-wave amplitudes (e0105, e0163), unusual shape of lead $\mathrm{V}_{4}$ signal (e0125), and a lot of high-frequency noise (e0139). Many of the false

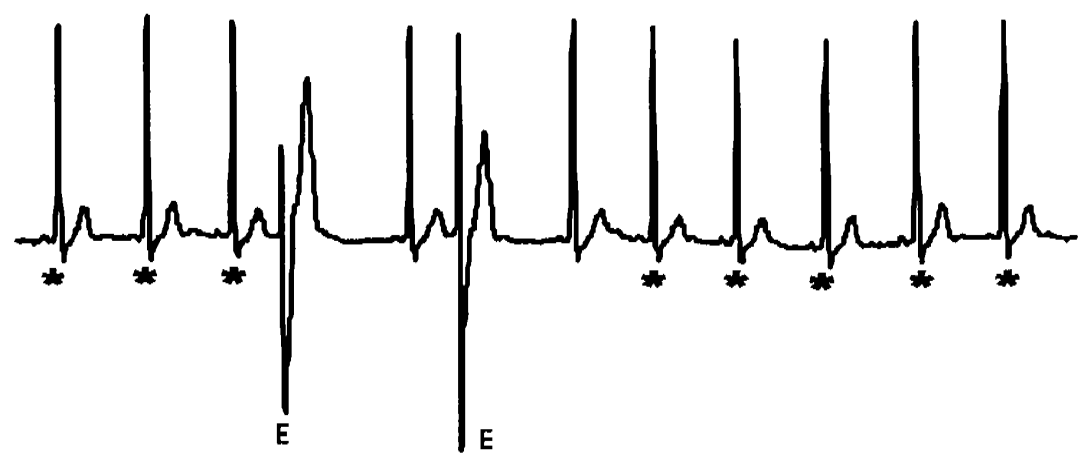

Figure 3: Delivery of EP pulses. The asterisks indicate when EP pulses would be delivered in real application. Absence of pulses at two extrasystoles (E) and at two normal QRS complexes following them demonstrates the ability of the algorithm to prevent the EP pulses from being delivered in case of abnormalilties in ECG shape or heart rate. 
Table 1: The results of evaluation of the QRS detection algorithm for signals from the ESC DB.

\begin{tabular}{cccccccc}
\hline Record & $\mathbf{N}$ & $\mathbf{T P}$ & FP & FN & Se(\%) & +P(\%) & DER(\%) \\
\hline $\mathrm{e} 0103$ & 7272 & 7182 & 45 & 90 & 98.76 & 99.38 & 1.86 \\
$\mathrm{e} 0105$ & 6600 & 5330 & 84 & 1270 & 80.76 & 98.45 & 20.52 \\
$\mathrm{e} 0113$ & 8915 & 8625 & 219 & 290 & 96.75 & 97.52 & 5.71 \\
$\mathrm{e} 0119$ & 7689 & 7175 & 93 & 514 & 93.32 & 98.72 & 7.89 \\
$\mathrm{e} 0121$ & 10594 & 10274 & 141 & 320 & 96.98 & 98.65 & 4.35 \\
$\mathrm{e} 0123$ & 9140 & 8833 & 40 & 307 & 96.64 & 99.55 & 3.80 \\
$\mathrm{e} 0125$ & 9031 & 7647 & 65 & 1384 & 84.68 & 99.16 & 16.04 \\
$\mathrm{e} 0127$ & 9358 & 8894 & 96 & 464 & 95.04 & 98.93 & 5.98 \\
$\mathrm{e} 0139$ & 10595 & 9051 & 215 & 1544 & 85.43 & 97.68 & 16.60 \\
$\mathrm{e} 0159$ & 9166 & 8533 & 360 & 633 & 93.09 & 95.95 & 10.83 \\
$\mathrm{e} 0163$ & 7580 & 6728 & 93 & 852 & 88.76 & 98.64 & 12.47 \\
$\mathrm{e} 0501$ & 7722 & 7365 & 147 & 357 & 95.38 & 98.04 & 6.53 \\
$\mathrm{e} 0607$ & 10233 & 9686 & 162 & 547 & 94.65 & 98.35 & 6.93 \\
\hline TOTAL & $\mathbf{1 1 3 8 9 5}$ & $\mathbf{1 0 5 3 2 3}$ & $\mathbf{1 7 6 0}$ & $\mathbf{8 5 7 2}$ & $\mathbf{9 2 . 4 7 ^ { \# }}$ & $\mathbf{9 8 . 3 6}$ & $\mathbf{9 . 0 7}$ \\
\hline
\end{tabular}

" Mean average values

Table 2: The results of evaluation of the EP pulse delivery algorithm for signals from the ESC DB.

\begin{tabular}{cccccccc}
\hline Record & $\mathbf{N}_{\mathbf{p}}$ & $\mathbf{T P}_{\mathbf{p}}$ & $\mathbf{F P}_{\mathbf{p}}$ & $\mathbf{F N}_{\mathbf{p}}$ & $\mathbf{S e}_{\mathbf{p}}(\mathbf{\%})$ & $\boldsymbol{+ P}_{\mathbf{p}}(\mathbf{\%})$ & $\mathbf{P D E R}(\mathbf{\%})$ \\
\hline $\mathrm{e} 0103$ & 7190 & 7077 & 0 & 113 & 98.43 & 100.00 & 1.57 \\
$\mathrm{e} 0105$ & 6598 & 4354 & 0 & 2244 & 65.99 & 100.00 & 34.01 \\
$\mathrm{e} 0113$ & 8897 & 8186 & 0 & 711 & 92.01 & 100.00 & 7.99 \\
$\mathrm{e} 0119$ & 7688 & 6870 & 1 & 818 & 89.36 & 99.99 & 10.65 \\
$\mathrm{e} 0121$ & 10579 & 10187 & 0 & 392 & 96.29 & 100.00 & 3.71 \\
$\mathrm{e} 0123$ & 9134 & 8672 & 0 & 462 & 94.94 & 100.00 & 5.06 \\
$\mathrm{e} 0125$ & 9027 & 6800 & 0 & 2227 & 75.33 & 100.00 & 24.67 \\
$\mathrm{e} 0127$ & 9358 & 8387 & 1 & 971 & 89.62 & 99.99 & 10.39 \\
$\mathrm{e} 0139$ & 10595 & 8382 & 0 & 2213 & 79.11 & 100.00 & 20.89 \\
$\mathrm{e} 0159$ & 9117 & 8407 & 0 & 710 & 92.21 & 100.00 & 7.79 \\
e0163 & 7567 & 5267 & 0 & 2300 & 69.60 & 100.00 & 30.40 \\
e0501 & 7717 & 7043 & 0 & 674 & 91.27 & 100.00 & 8.73 \\
e0607 & $\mathbf{1 0 1 8 5}$ & 9301 & 0 & 884 & 91.32 & 100.00 & 8.68 \\
\hline TOTAL & $\mathbf{1 1 3 6 5 2}$ & $\mathbf{9 8 9 3 3}$ & $\mathbf{2}$ & $\mathbf{1 4 7 1 9}$ & $\mathbf{8 7 . 0 5}$ & $\mathbf{1 0 0 . 0 0}$ & \multirow{2}{*}{$\mathbf{1 2 . 9 5}$} \\
\hline
\end{tabular}

\# Mean average values 
negative $(F N)$ detections were also due to very strict requirements imposed for positive QRS detection (subsection 2.1.3).

In the absence of standard performance measures for EP pulse delivery, we used measures similar to those defined for the QRS detection. These measures can serve for comparison of performance of different algorithms for EP pulse delivery. It should be noted that our main concern here was to avoid application of EP pulses at the moments of potential danger for the patient at any cost. The algorithm fulfilled this requirement excellently as indicated by practically ideal $+P_{p}$ values (Table 2 ). We made our algorithm deliberately more conservative than would be necessary if the purpose was solely to detect QRS complexes. It was completely acceptable to miss some normal heartbeats as long as no EP pulse was delivered when it absolutely should not be. Upon careful examination of the records in which the $F P_{p}$ was not zero we found that the erroneously delivered pulses (there were only two such pulses in comparison to approximately $10^{5}$ correctly delivered pulses) coincided with sudden changes in the signal characteristics, which were most probably caused by deterioration of the skin-electrodes interface during ECG recording. At this preliminary stage of the development our algorithm is not well suited to deal with signals that are largely nonstationary or have very low signal-to-noise ratio. This situation can be relatively easily improved by subjecting the threshold values in the QRS detector to some on-line adaptive algorithm, for example in a manner similar to the moving average evaluation of the normal current heart rate. It is important to note that due to its conservative nature the algorithm dealt well with mildly nonstationary parts of the signal or transient noise contamination, which were occasionally encountered in most of the signals used in algorithm evaluation.

Some abnormalities in functioning of the heart may not be distinguishable from a normal heartbeat by the present algorithm. If the EP pulses could aggravate the underlying physiological conditions, the algorithm should be supplemented with more elaborate decision rules for EP pulse delivery in the future.

\section{Conclusion}

The algorithm for on-line synchronization of EP pulse delivery with ECG would present a significant improvement over the existing practice of EP pulse delivery with respect to the safety of the patient. The proposed algorithm allows the EP pulses to be delivered only outside the vulnerable period of the heartbeat and prevents the EP pulses from being delivered when heart arrhythmias are present.

\section{Acknowledgement}

The presented work was performed for the purpose of two projects of $5^{\text {th }} \mathrm{EU}$ Framework Programme (CLINIPORATOR and ESOPE) within which instrumentation and procedures for clinical use of electrochemotherapy and gene electrotransfection are being developed and evaluated. 


\section{References}

[1] Mir, L.M., Glass, L.F., Sersa, G., Teissie, J., Domenge, C., Miklavcic, D., Jaroszeski, M.J., Orlowski, S., Reintgen, D.S., Rudolf, Z., Belehradek, M., Gilbert, R., Rols, M.P., Belehradek, J.Jr., Bachaud, J.M., DeConti, R., Stabuc, B., Cemazar, M., Coninx, P. \& Heller, R., Effective treatment of cutaneous and subcutaneous malignant tumors by electrochemotherapy. Brit $J$ Cancer, 77, pp. 2336-2342, 1998.

[2] Heller, R., Jaroszeski, M.J., Reintgen, D.S., Puleo, C.A., DeConti, R.C., Gilbert, R.A. \& Glass, L.F., treatment of cutaneous and subcutaneous tumors with electrochemotherapy using intralesional bleomycin. Cancer, 83, pp. 148-157, 1998.

[3] Sersa, G., Stabuc, B., Cemazar, M., Miklavcic, D. \& Rudolf, Z., Electrochemotherapy with cisplatin: Clinical experience in malignant melanoma patients. Clin Cancer Res, 6, pp. 863-867, 2000.

[4] Sersa, G., Cufer, T., Cemazar, M., Miklavcic, D., Rebersek, M. \& Rudolf, Z., Electrochemotherapy with bleomycin in the treatment of hypernephroma metastasis: case report and literature review. Tumori, 86, 163-165, 2000.

[5] Mir, L.M., Orlowski, S., Belehradek, J.Jr. \& Paoletti, C., Electrochemotherapy potentiation of antitumor effect of bleomycin by local electric pulses. Eur J Cancer, 27, pp. 68-72, 1991.

[6] Mir, L.M. \& Orlowski, S., Mechanisms of electrochemotherapy. Adv Drug Delivery Rev, 35, pp. 107-118, 1999.

[7] Reilly, J.P., Applied bioelectricity: from electrical stimulation to electropathology, Springer-Verlag: New York, pp. 148-239, 1998.

[8] Berne, R.M., Levy, M.N., Koeppen, B.M. \& Stanton, B.A., Physiology, Mosby: St, Louis, pp. 329-359, 1998.

[9] Friesen, G.M., Jannett, T.C., Jadallah, M.A., Yates, S.L., Quint, S.R. \& Nagle, H.T., A comparison of the noise sensitivity of nine QRS detection algorithms. IEEE Trans Biomed Eng, 37, pp. 85-98, 1990

[10] Pan, J. \& Tompkins, W.J., A real-time QRS detection algorithm. IEEE Trans Biomed Eng, 32(3), pp. 230-236, 1985.

[11] Hamilton, P.S. \& Tompkins W.J., Quantitative investigation of QRS detection rules using the MIT/BIH Arrhythmia Database. IEEE Trans Biomed Eng, 33(12), pp. 1157-1165, 1986.

[12] Laguna, P., Jané, R. \& Caminal, P., Automatic detection of wave boundaries in multilead ECG signals: Validation with the CSE Database. Comput Biomed Res, 27, pp. 45-60, 1994.

[13] Taddei, A., Distante, G., Emdin, M., Pisani, P., Moody, G.B., Zeelenberg, C. \& Marchesi, C., The European ST-T Database: standard for evaluating systems for the analysis of ST-T changes in ambulatory electrocardiography. Eur Heart J, 13, pp. 1164-1172, 1992.

[14] http://www.physionet.org/physiobank/database/edb/.

[15] Benitez, D.S., Gaydecki, P.A., Zaidi, A. \& Fitzpatrick, A.P. Laguna, P., A new QRS detection algorithm based on the Hilbert transform. Computers in Cardiology, 27, pp. 379-382, 2000. 